\title{
BMJ Open Impact of levothyroxine in women with positive thyroid antibodies on pregnancy outcomes: a systematic review and meta-analysis of randomised controlled trials
}

\author{
Lorraine Lau (D) , ${ }^{1}$ Jamie L Benham, ${ }^{1,2}$ Patricia Lemieux, ${ }^{1}$ Jennifer Yamamoto, ${ }^{1,3,4,5}$ \\ Lois E Donovan ${ }^{1,3,4}$
}

To cite: Lau L, Benham JL, Lemieux P, et al. Impact of levothyroxine in women with positive thyroid antibodies on pregnancy outcomes: a systematic review and meta-analysis of randomised controlled trials. BMJ Open 2021;11:e043751. doi:10.1136/ bmjopen-2020-043751

- Prepublication history and additional material for this paper is available online. To view these files, please visit the journal online (http://dx.doi.org/10. 1136/bmjopen-2020-043751)

Received 17 August 2020 Revised 19 December 2020 Accepted 01 February 2021

D Check for updates

(c) Author(s) (or their employer(s)) 2021. Re-use permitted under CC BY-NC. No commercial re-use. See rights and permissions. Published by BMJ.

For numbered affiliations see end of article.

Correspondence to Dr Lois E Donovan;

lois.donovan@albertahealthser vices.ca

\section{ABSTRACT}

Objective To evaluate the effect of levothyroxine therapy on pregnancy outcomes compared with placebo or no treatment in women without overt hypothyroidism with presence of thyroid peroxidase antibodies (TPOAb) and/or thyroglobulin antibodies (TgAb).

Design Systematic review and meta-analysis of randomised controlled trials

Study eligibility criteria Prespecified criteria for inclusion were: randomised trials of levothyroxine versus control (placebo or no treatment) among women with positive TPOAb or TgAb who were pregnant or considering conception.

Data sources Ovid MEDLINE, EMBASE, CINAHL, Cochrane Database of Systematic Reviews and Cochrane Central Register of Controlled Trials were searched from 1980 to 5 November 2020.

Outcome measures Prespecified data elements were extracted and where appropriate, meta-analyses were conducted. Main outcomes include pregnancy achieved, miscarriage, preterm delivery and live birth.

Risk of bias assessment Cochrane Risk of Bias Tool for Quality Assessment of Randomised Controlled Trials. Results From 3023 citations, 79 citations were identified for full-text review. Of these, six trials (total of 2263 women) were included for qualitative and quantitative analyses. Risk of bias was deemed low for only one trial. There was no significant difference in the relative risk (RR) of pregnancy achieved (RR 1.03; 95\% Cl 0.93 to 1.13), miscarriage (RR $0.93 ; 95 \% \mathrm{Cl} 0.76$ to 1.14 ), preterm delivery (RR $0.66 ; 95 \% \mathrm{Cl} 0.39$ to 1.10 ) or live births (RR $1.01 ; 95 \% \mathrm{Cl} 0.89$ to 1.16 ) in thyroid autoimmune women treated with levothyroxine compared with controls. Sensitivity analyses of preterm birth identified study quality and timing of levothyroxine initiation as sources of heterogeneity.

Conclusions Among pregnant women or women planning conception, with thyroid autoimmunity, there is a lack of evidence of benefit for levothyroxine use (moderate to high Grading of Recommendations, Assessment, Development and Evaluations). Recommendations to use levothyroxine in this setting need to be reconsidered.

PROSPERO registration number CRD42019130459.
Strengths and limitations of this study

- This systematic review summarises the most current published randomised controlled trials not included in other systematic reviews and meta-analysis.

- This systematic review and meta-analysis is rigorous in design, methodology and analyses with a prepublished protocol.

Of the six trials identified for inclusion, only one was deemed low risk of bias.

There was evidence of publication bias.

\section{INTRODUCTION}

As thyroid function is frequently screened during the early gestational period, identification of thyroid immunity, defined by the presence of thyroid peroxidase antibodies (TPOAb) and/or thyroglobulin antibodies (TgAb), is relatively common in pregnant women. The prevalence of TPOAb positivity in the first trimester ranges from $5 \%$ to $15 \%$ in unselected pregnant women. ${ }^{12}$ The prevalence of thyroid autoimmunity is even higher in women with a history of recurrent pregnancy loss, reported as $23 \%-31 \% .^{34}$

The association between thyroid autoimmunity and poor pregnancy outcomes, specifically miscarriage and preterm birth, has been well described ${ }^{5}{ }^{6}$ However, the mechanism linking thyroid autoimmunity with increased risk of miscarriage and preterm birth in women without overt hypothyroidism is unclear. Two competing mechanistic theories propose that (1) thyroid autoantibodies are a marker of a hostile immune environment or (2) thyroid autoantibodies act as a surrogate biomarker of impaired thyroid hormone reserves, despite normal thyroid function tests. ${ }^{7}$ If the latter theory is true, one could postulate that supplementation with 
levothyroxine may improve thyroid hormone levels and prevent negative pregnancy outcomes.

Whether or not levothyroxine therapy, either prior to or during pregnancy, improves pregnancy outcomes in women with thyroid autoimmunity is controversial. ${ }^{8-14}$ The 2017 American Thyroid Association (ATA) pregnancy guidelines strongly recommends levothyroxine for women with thyroid autoimmunity and a thyroid stimulated hormone (TSH) above the pregnancy-specific reference range. These guidelines recommend a consideration of levothyroxine in thyroid autoimmune women with TSH above $2.5 \mathrm{mIU} / \mathrm{L}$ and among euthyroid women with positive TPOAb and a history of pregnancy loss. ${ }^{15}$ The evidence evaluating levothyroxine treatment in pregnant women with thyroid autoantibodies is conflicting as evidenced by three recent systematic reviews and metaanalyses. ${ }^{13} 1617$ The discrepant results may be attributed to the inclusion of small observational studies and the lack of inclusion of a recently published large multicentre trial. ${ }^{8}$ Additionally, the limitations of observational studies must be considered. Observational studies identify associations, but do not provide the highest level of evidence for determining causation or therapeutic efficacy. Randomised controlled trials are considered the gold standard to evaluate therapeutic efficacy. ${ }^{18}$ Therefore, we performed a systematic review and meta-analysis to evaluate all randomised controlled trials of levothyroxine therapy in women with thyroid autoimmunity who were pregnant or planning pregnancy.

\section{METHODS}

\section{Protocol and registration}

A systematic review and meta-analysis was performed as outlined in the registered protocol (PROSPERO CRD42019130459), and is reported in accordance with the Preferred Reporting Items for Reviews and Meta-Analyses.

\section{Data sources and search strategy}

The following databases were searched in duplicate on 17 April 2019 (and updated on 5 November 2020) from 1980: Ovid MEDLINE, EMBASE, CINAHL, Cochrane Database of Systematic Reviews and Cochrane Central Register of Controlled Trials. ClinicalTrials.gov was searched for active trials. The search strategy was developed in consultation with a medical librarian and used a combination of keywords related to hypothyroidism or thyroid autoimmunity and pregnancy (online supplemental S1). The search was conducted using an adapted version of the Cochrane search filter for randomised controlled trials where possible and was limited to human studies. ${ }^{19}$

\section{Study selection}

Studies that met all the following criteria were included in this review: (1) pregnant women or women contemplating pregnancy (including patients seeking assisted reproductive technologies), with positive TPOAb or TgAb, euthyroid or with subclinical hypothyroidism (by any prespecified study definition); (2) randomised to levothyroxine vs control (placebo or no treatment); (3) reported pregnancy outcomes; (4) reported in either English or French; (5) published from 1980 to 5 November 2020. The publication year 1980 was specifically chosen to coincide with the availability of more sensitive TSH assays. ${ }^{201}$

Study selection was completed in two separate stages by two independent reviewers (PL and LED or LL and JLB). First, duplicates were removed, and titles and abstracts were screened for eligibility (by PL and LED). Second, full citations deemed eligible at the title and abstract stage were retrieved and reviewed for eligibility in a full-text format (by LL and JLB). Inclusion criteria was applied to select eligible articles and reasons for exclusion were recorded. Agreement was recorded at each stage and reported as a kappa statistic. ${ }^{22}$ All disagreements between reviewers were solved by consensus, and, if needed, by discussion with a third independent reviewer (LED). Prior to each selection step, a pilot calibration was conducted by the respective reviewers to review inclusion criteria. The reference list of included articles was searched for the addition of further relevant studies.

\section{Data collection process}

Relevant study information was extracted by two independent reviewers (LL and JB) using standardised data extraction forms in Microsoft Excel (V.16.16.18, Microsoft Corporation, Redmon, Washington, USA). Extracted data elements included: study design, country, participant inclusion and exclusion criteria, TPOAb and TgAb results, definition of TPOAb and TgAb positivity, TPOAb and $\mathrm{TgAb}$ assays used, thyroid stimulating hormone (TSH) and thyroxine (T4) assays used and normal ranges, baseline serum concentrations of TSH and T4, iodine status of the country, levothyroxine dosing, achieved TSH and T4 after treatment, gestational timing of levothyroxine initiation, duration of treatment, details of the intervention, quality of the methods, inclusion of patients with subclinical hypothyroidism and definition of subclinical hypothyroidism, patient baseline characteristics, number of patients included in each group, pregnancy outcomes and their definitions.

\section{Quality assessment}

Cochrane Risk of Bias Tool for Quality Assessment of Randomised Controlled Trials ${ }^{23}$ was used to assess study quality and evaluate the risk of bias by two independent reviewers (LL and JLB). This tool assesses seven methodologic domains and these components enable classification of randomised trials as 'low risk of bias', 'high risk of bias' or 'unclear risk of bias'.

\section{Study outcomes}

The following pregnancy outcomes were prespecified and included: pregnancy by trial definition, miscarriage, preterm delivery, live birth, birth weight, large for gestational age, small for gestational age, gestational age at 
delivery, multiple pregnancy, stillbirth, neonatal intensive care unit admission, pre-eclampsia and gestational diabetes. Grading of Recommendations, Assessment, Development and Evaluations (GRADE) was used for grading of quality of evidence for the main outcomes. ${ }^{24}$

\section{Data synthesis and analysis}

Where appropriate, meta-analyses were conducted to evaluate the relative risk (RR) and 95\% CI of prespecified pregnancy outcomes including pregnancy achieved, miscarriage, preterm delivery and live birth in participants randomised to levothyroxine therapy compared with placebo or no treatment. Random effects models were used. Statistical heterogeneity was quantified using $\mathrm{I}^{2}$ statistic, where $\mathrm{I}^{2}>50 \%$ represents moderate and $\mathrm{I}^{2}>75 \%$ represents substantial heterogeneity across studies. ${ }^{25}$ The following prespecified sensitivity analyses were conducted to explore heterogeneity: (1) TSH levels at baseline, (2) time of levothyroxine initiation (preconception or during pregnancy), (3) use and type of assisted reproduction technologies, (4) maternal characteristics and (5) study quality. Publication bias was assessed using a funnel plot as the graphical aid. Statistical analysis was performed using STATA V.14 (StataCorp) and Review Manager V.5.3 (V.5.3.5, The Cochrane Collaboration, Copenhagen, Denmark).

\section{Patient and public involvement}

No patient involved.

\section{RESULTS}

The search results are summarised in figure 1. Among the 3023 citations reviewed for title and abstract, 79 citations were identified for full-text review. Six randomised

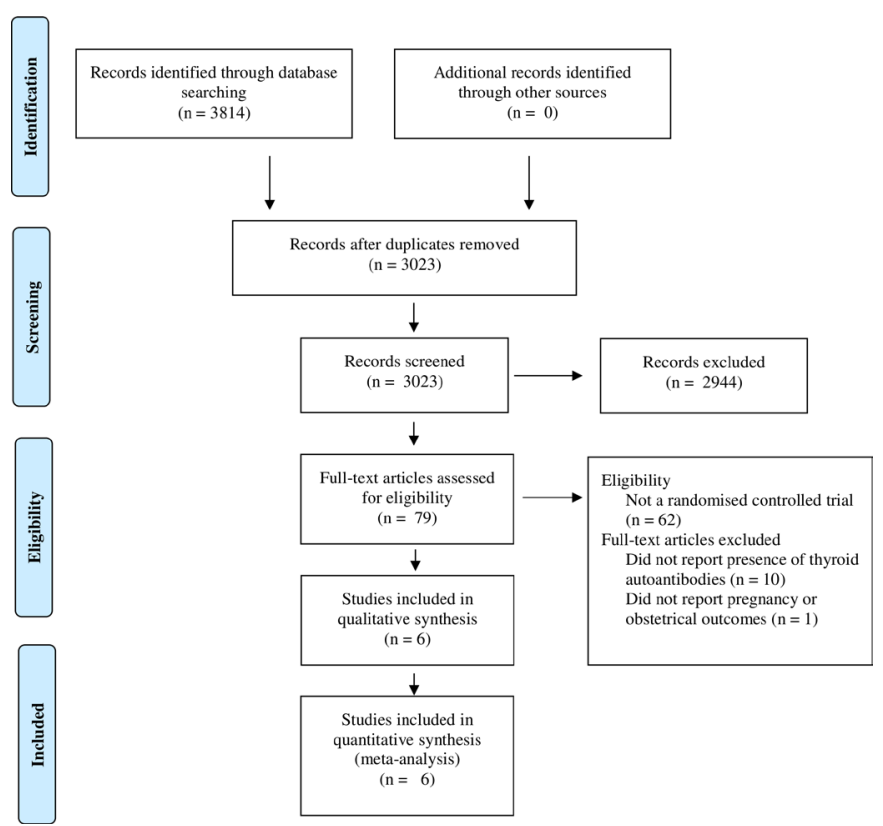

Figure 1 Preferred Reporting Items for Systematic Reviews and Meta-Analyses flow diagram. controlled trials (total of 2263 women) met all eligibility criteria and were included in the qualitative and quantitative analyses. ${ }^{8-12} 14$ There was a substantial level of agreement for both the title and abstract review $(\kappa=0.64)$ and for the full-text review $(\kappa=0.70)$.

\section{Trial characteristics}

Trial characteristics are summarised in table 1. Three trials initiated levothyroxine therapy prior to conception, ${ }^{81114}$ whereas three trials initiated therapy in the first trimester. ${ }^{91012}$ Two trials compared levothyroxine to placebo, ${ }^{810}$ and the other four trials compared with no treatment. ${ }^{911} 1214$ Reproductive technologies were used in three trials, ${ }^{811} 14$ with one trial not specifying details with this regard. All trials included women with positive TPOAb with only one trial including TPO or Tg antibodies. ${ }^{14}$ The TSH normal reference ranges and definition of TPO positivity were prespecified in each trial and are summarised in table 1 .

\section{Patient characteristics}

Baseline participant characteristics are summarised in table 1 and online supplemental S2. Mean maternal age ranged from 26 years ${ }^{9}$ to 32 years. ${ }^{8}$ Mean reported body mass index ranged from $22.7 \mathrm{~kg} / \mathrm{m}^{2}{ }^{14}$ to 26.5 $\mathrm{kg} / \mathrm{m}^{2}{ }^{8}$ Baseline TSH values varied according to assay specific reference ranges. Baseline-free T4 values were comparable in the three trials that reported this information $^{811} 12$ (online supplemental S2). There was insufficient information to assess the outcome of childhood neurodevelopment.

\section{Risk of bias assessment}

Risk of bias assessment is presented in figure 2. Only one trial $^{8}$ was considered 'low risk' of bias in all seven domains. Four trials ${ }^{810-12}$ were assessed at 'low risk' for selection bias. Two trials ${ }^{914}$ were 'unclear risk of bias' in allocation concealment. In four trials comparing levothyroxine to no treatment, ${ }^{9101214}$ the assessors were not blinded to patient randomisation and thus these trials were considered to be 'high risk' for performance bias. Published trial protocols were located for only two trials. ${ }^{8}{ }^{14}$ Thus, only these two trials were assessed as 'low risk' for reporting bias.

\section{Pregnancy outcomes}

There were sufficient data for meta-analyses of four pregnancy outcomes, as summarised in figure 3 . These outcomes include pregnancy achieved, miscarriage, preterm delivery and live births.

Pregnancy achieved was reported in three trials $^{81114}(\mathrm{n}=1626)$ that initiated levothyroxine preconception. There was no significant difference in pregnancy achieved in thyroid autoimmune women treated levothyroxine compared with control (RR 1.03, 95\% CI 0.93 to $\left.1.13 ; \mathrm{I}^{2}=0 \%\right)$. GRADE was rated as high quality for this outcome (table 2).

Miscarriage was reported in all six trials $(\mathrm{n}=1427)$ and was defined as pregnancy loss at less than 20 weeks, less 

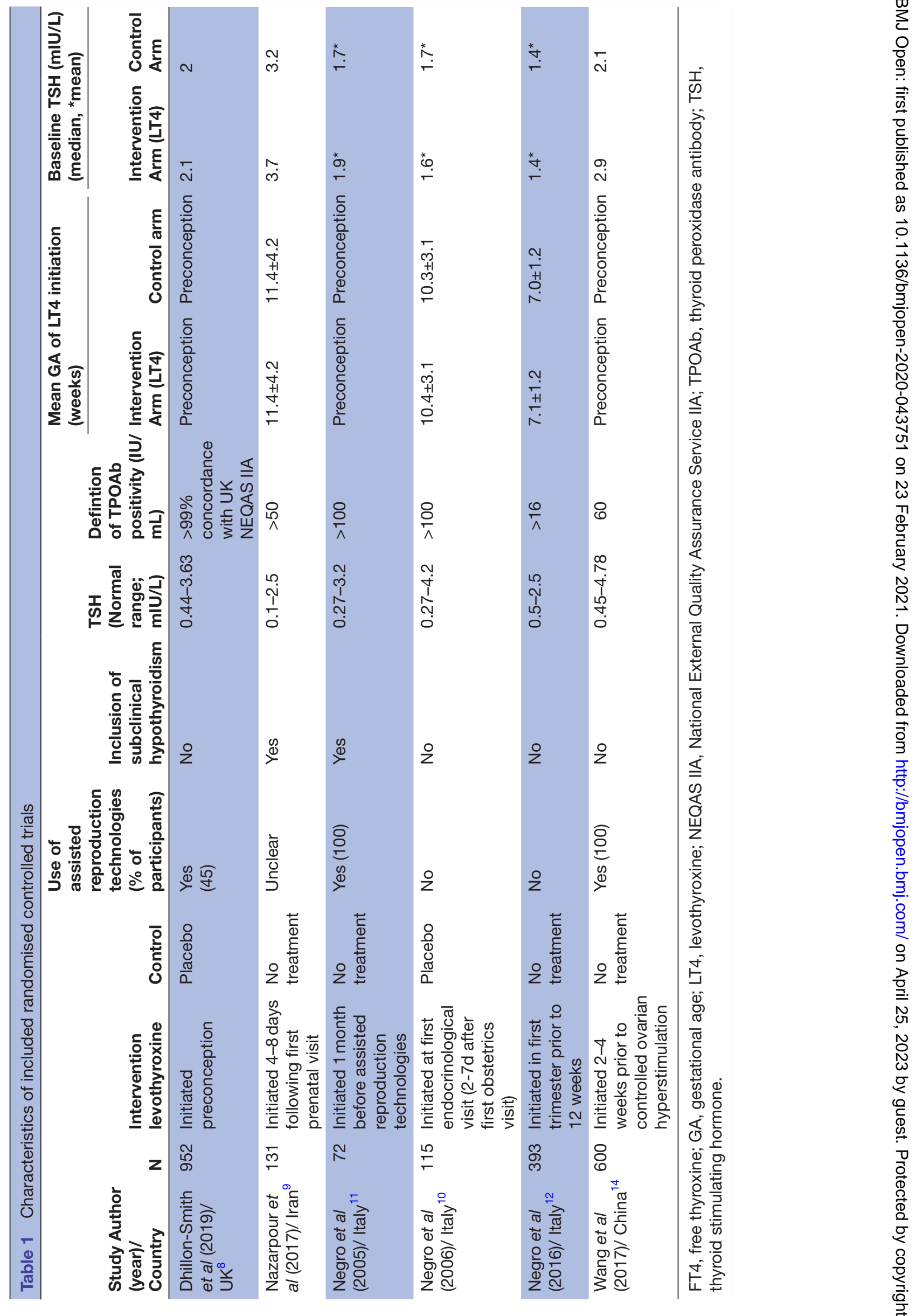

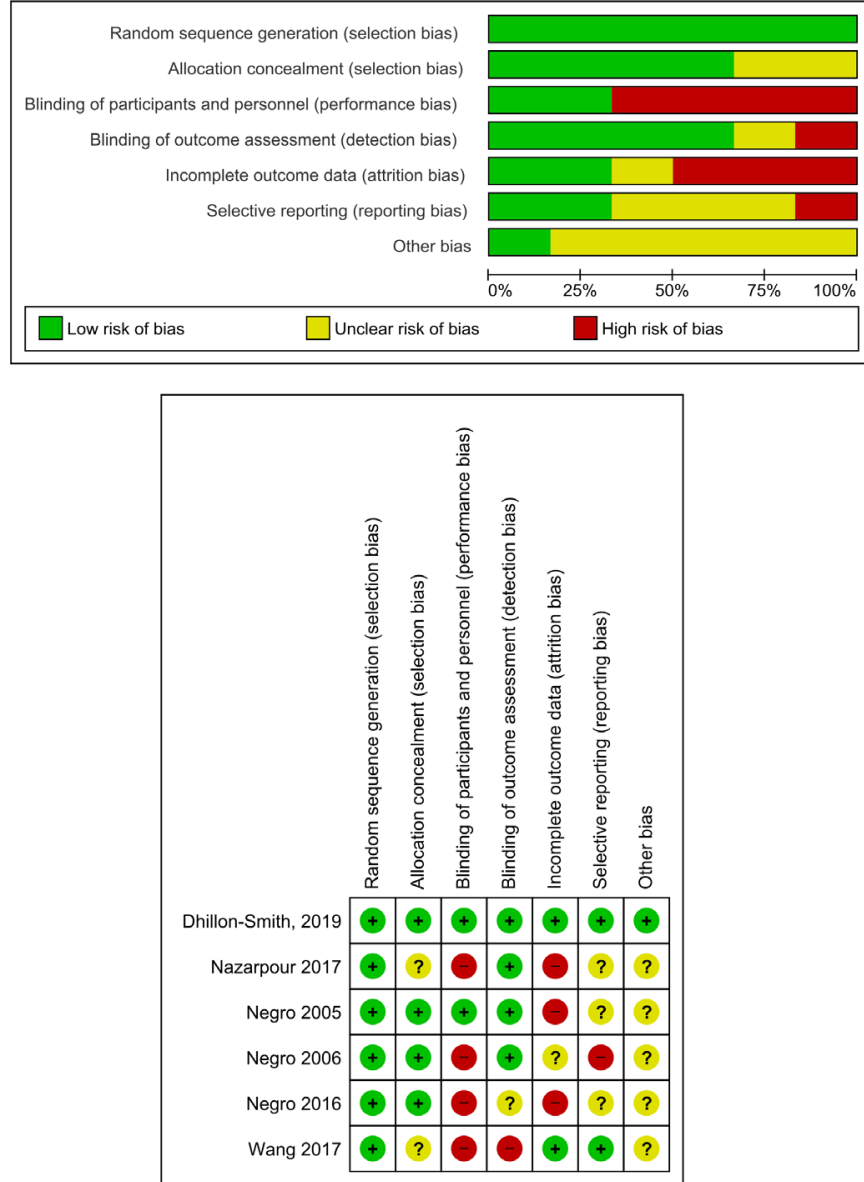

Figure 2 Risk of bias assessment and trial quality assessment.

than 24 weeks, less than 28 weeks (early miscarriage defined as first 12 weeks, late miscarriage defined as between 13 and 28 weeks), ${ }^{14}$ or not clearly defined. ${ }^{10-12}$ There was no significant difference in the RR of miscarriage in TPOAb-positive pregnant women treated with levothyroxine compared with control (RR 0.93, 95\% CI 0.76 to $\left.1.14 ; \mathrm{I}^{2}=0 \%\right)$. Inclusion of trials ${ }^{811} 14$ that initiated levothyroxine only preconception revealed no significant difference compared with control (RR $0.91,95 \%$ CI 0.72 to $1.15 ; \mathrm{I}^{2}=0 \%$ ). GRADE was rated as high quality for this outcome (table 2).

Preterm delivery was reported in five trials $(\mathrm{n}=1354){ }^{8-1012} 14$ Preterm delivery was defined as live birth prior to 34 weeks $^{8}$ or 37 weeks. ${ }^{9} 101214$ There was no significant difference in the RR of preterm delivery in thyroid autoimmune pregnant women treated with levothyroxine compared with control (RR 0.66 , 95\% CI 0.39 to $1.10 ; \mathrm{I}^{2}=53 \%$ ). GRADE was rated at moderate quality for this outcome. Downgrade considerations include considerable inconsistency, wide CIs contributing to imprecision and evidence of publication bias.

Live births were reported in two trials $(\mathrm{n}=1540) .{ }^{8}{ }^{14}$ Live birth was defined as delivery of a living fetus beyond 27 weeks ${ }^{14}$ or beyond 34 weeks. ${ }^{8}$ There was no significant difference in live births in thyroid autoimmune women treated with levothyroxine compared with control (RR
$1.01,95 \%$ CI 0.89 to $\left.1.16 ; \mathrm{I}^{2}=0 \%\right)$. GRADE was rated at high quality for this outcome (table 2).

Only one trial ${ }^{10}$ reported on pre-eclampsia and found no difference in this outcome among thyroid autoimmune women treated with levothyroxine compared with control. None of the studies reported the outcome of gestational diabetes.

\section{Neonatal outcomes}

Neonatal outcomes were only reported in two trials $(\mathrm{n}=1083) .{ }^{8}$ Meta-analysis for gestational age at time of delivery (measured in days) and birth weight (measured in grams) are summarised in online supplemental S3. The weighted mean difference for gestational age at time of delivery in women treated with levothyroxine compared with placebo or no treatment was 2.35 days (95\% CI -4.51 to $\left.9.20 ; \mathrm{I}^{2}=90 \%\right)$. Weighted mean difference for birth weight in women treated with levothyroxine compared with placebo or no treatment was $-24.05 \mathrm{~g}$ (95\% CI -96.91 to $48.80 ; \mathrm{I}^{2}=0 \%$ ).

Only one trial ${ }^{9}$ reported neonatal intensive care unit admission. They found fewer admissions among thyroid autoimmune women treated with levothyroxine (2 of 56 compared with control 12 of $58, \mathrm{p}<0.05)$. No studies reported large for gestational age, small for gestational age or multiple pregnancy.

\section{Sensitivity analyses}

A sensitivity analysis was performed to explore the heterogeneity of our meta-analysis for preterm delivery. Results are summarised in online supplemental S4.

TSH levels at baseline were subdivided into TSH $\leq 2.5$ $\mathrm{mIU} / \mathrm{mL}^{81012}$ and $\mathrm{TSH}>2.5 \mathrm{mIU} / \mathrm{mL}^{9}{ }^{14}$ The $\mathrm{RR}$ of preterm delivery in pregnant women with baseline TSH $\leq 2.5 \mathrm{mIU} / \mathrm{mL}$ was $0.64\left(95 \%\right.$ CI 0.36 to $1.14 ; \mathrm{I}^{2}=32 \%$ ) compared with an RR of 0.62 (95\% CI 0.17 to 2.33; $\mathrm{I}^{2}=80 \%$ ) in pregnant women with baseline TSH $>2.5$ $\mathrm{mIU} / \mathrm{mL}$.

Timing of levothyroxine initiation was stratified into preconception ${ }^{814}$ and in pregnancy. ${ }^{91012}$ The RR of preterm delivery in women initiated on levothyroxine preconception was 1.10 (95\% CI 0.69 to 1.75 ; $\mathrm{I}^{2}=0 \%$ ) compared an RR of 0.46 (95\% CI 0.28 to 0.75 ; $\left.\mathrm{I}^{2}=2 \%\right)$ in women initiated on levothyroxine in the first trimester.

The RR of preterm delivery in women using assisted reproductive technologies (ART) $)^{814}$ was $1.10(95 \%$ CI 0.69 to $1.75, \mathrm{I}^{2}=0 \%$ ) compared with an $\mathrm{RR}$ of 0.51 ( $95 \%$ CI 0.26 to $1.02 ; \mathrm{I}^{2}=27 \%$ ) in women that did not use ART. ${ }^{1012}$

Sensitivity analyses were also performed to explore the impact of fixed ${ }^{811}$ and adjusted levothyroxine ${ }^{9101214}$ doses on miscarriage rates (online supplemental S5). The RR of fixed levothyroxine dose was 0.89 (95\% CI 0.66 to 1.20 ; $\mathrm{I}^{2}=12 \%$ ) compared with adjusted levothyroxine dose of 0.95 (95\% CI 0.68 to $\left.1.33 ; \mathrm{I}^{2}=0 \%\right)$.

Study quality was defined as high if there was low risk of bias across all seven domains of the Cochrane 
A Pregnancy achieved

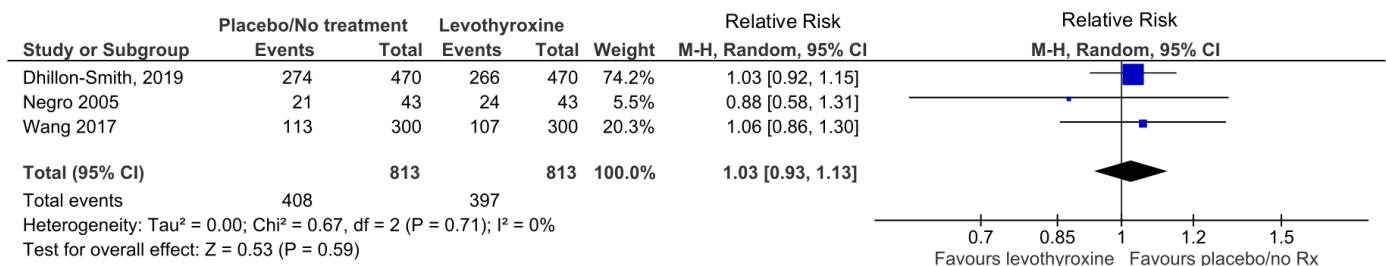

B Miscarriage

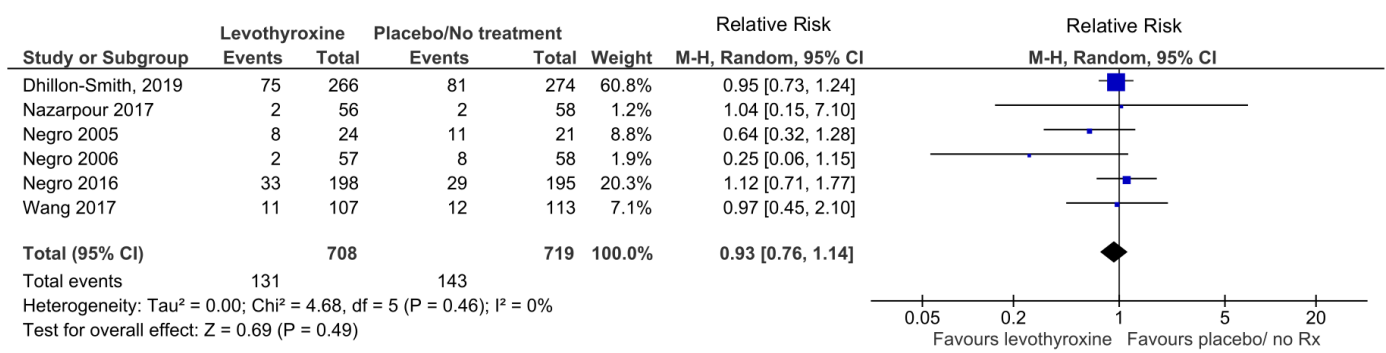

C Preterm delivery

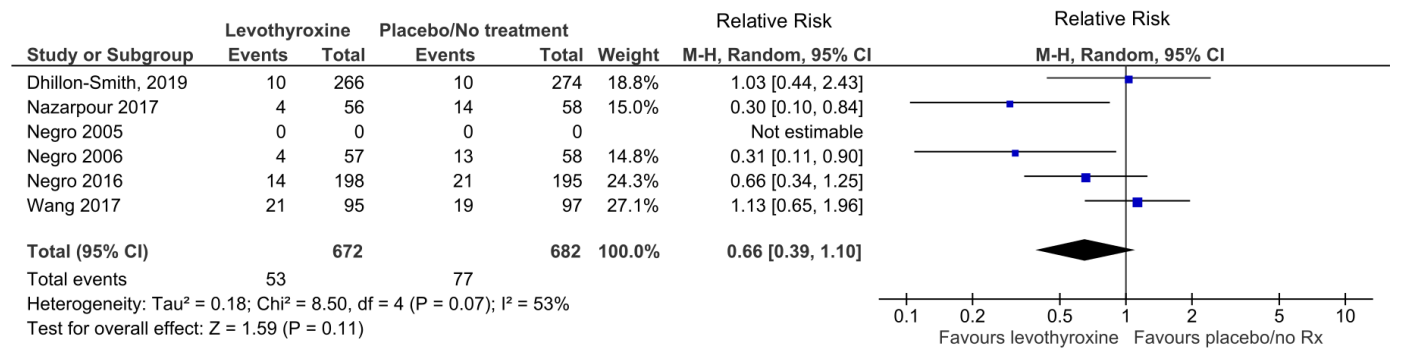

D Live births

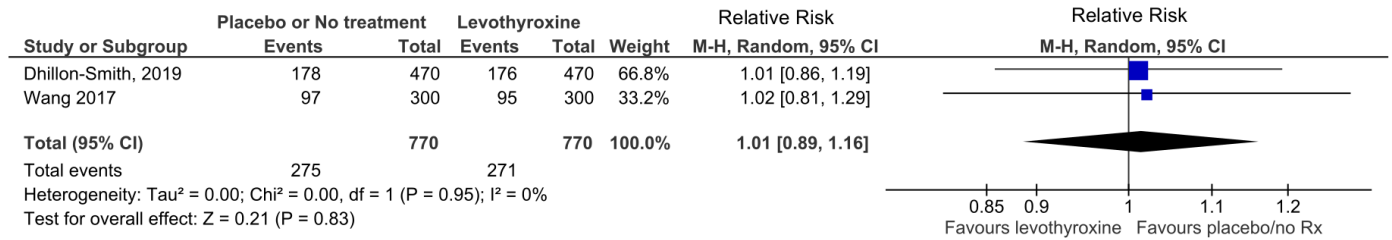

Figure 3 Results of meta-analysis of effects of levothyroxine treatment on pregnancy and obstetrical outcomes. M- $\mathrm{H}$, MantelHaenszel.

Collaboration's tool. We were unable to perform a sensitivity analysis exploring the impact of risk of bias on preterm delivery, as only one trial met the definition for high quality per the Cochrane Collaboration tool. ${ }^{8}$
A funnel plot for preterm delivery is shown in online supplemental S6. On visual inspection, there was asymmetry indicating potential publication bias towards smaller studies reporting positive findings.

Table 2 Grading of Recommendations, Assessment, Development and Evaluations (GRADE) scoring of the quality of evidence for the main outcomes

\begin{tabular}{|c|c|c|c|c|}
\hline Outcome & Pregnancy achieved & Miscarriage & Preterm delivery & Live births \\
\hline Risk of bias & Strong & Strong & Moderate & Strong \\
\hline Imprecision & Strong & Strong & Moderate & Strong \\
\hline Indirectness & Strong & Strong & Strong & Strong \\
\hline Publication bias & Strong & Strong & Weak & Strong \\
\hline
\end{tabular}




\section{DISCUSSION}

This systematic review and meta-analysis of randomised controlled trials of levothyroxine therapy in women with thyroid autoimmunity without overt thyroid dysfunction demonstrates no evidence of benefit of levothyroxine therapy across prespecified pregnancy outcomes. Specifically, treatment with levothyroxine did not improve pregnancies achieved, miscarriage preterm delivery or live birth across a total of 2263 women, inclusive of those with a history of pregnancy loss and use of reproductive technologies. Furthermore, the availability of larger and better-quality trials since the publications of the ATA 2017 pregnancy recommendations signals the need to update these guidelines as they pertain to the treatment of thyroid autoimmune women.

Small randomised clinical trials initially demonstrated that levothyroxine therapy reduced miscarriage and/or preterm delivery in TPOAb-positive women compared with placebo or no treatment intervention. ${ }^{9-11}$ However, the unclear definition of miscarriage and preterm delivery among these early trials means that selective reporting of outcomes could have biased their reported findings. Subsequent large, multi-centre randomised controlled trials with clear prespecified outcome definitions have shown no impact of levothyroxine therapy on maternal or fetal outcomes compared with no levothyroxine treatment in pregnant women with subclinical hypothyroidism, isolated hypothyroxinaemia or positive TPOAb. ${ }^{8} 142627$ These intervention trials involved pregnant women or women seeking pregnancy, including individuals with a history of pregnancy loss.

A systematic review and meta-analysis of a large study population $(n=47045)$ of individual participant and population data demonstrated increased risk of preterm birth in TPOAb-positive women. ${ }^{6}$ In light of these findings, Cappola and Case ${ }^{28}$ highlighted the need to address whether levothyroxine treatment decreases the risk of preterm birth among women with TPOAb. Three other systematic reviews and meta-analyses examined the effect of levothyroxine supplementation in pregnant women with thyroid autoimmunity. ${ }^{13} 1617$ Unlike our review which only included randomised controlled trials, two of the other reviews ${ }^{24} 25$ included observational studies. The findings of our meta-analysis were consistent with the review by Sun $e t a l,{ }^{17}$ which showed no benefit of levothyroxine supplementation on their pregnancy outcomes. In contrast, Rao et al $^{1316}$ demonstrated a beneficial effect of levothyroxine treatment on reducing pregnancy loss and preterm birth among pregnant women with subclinical hypothyroidism and/or thyroid autoimmunity. Several notable distinctions likely account for these discrepant results. First, the Dhillon-Smith $e t a l^{8}$ publication was not included in these reviews. Furthermore, both reviews by Rao $e a^{1316}$ included a trial that strongly influenced their results but did not report outcome data in sufficient detail to be included in our systematic review. ${ }^{29}$ Our attempts to clarify the outcome data of this trial ${ }^{29}$ with the authors were left unresponded.
The findings of our study are consistent with the results of a recent systematic review and meta-analysis of randomised controlled trials of women with positive TPO antibodies. ${ }^{30}$ These authors also conclude that levothyroxine supplementation is not associated with improvement in pregnancy outcomes. This further emphasises the need to reconsider the current 2017 ATA guidelines on use of levothyroxine in euthyroid women with thyroid autoimmunity.

Most miscarriages occur early in pregnancy, prior to the first obstetrics appointment, thus initiation of levothyroxine prior to conception best addresses its impact on miscarriage rates. The pathophysiology underlying the association between thyroid autoimmunity in euthyroid pregnant women and miscarriage is still not completely understood. One hypothesis postulates that thyroid autoimmunity reflects inadequate thyroid reserves to accommodate for increased physiologic demands of pregnancy. ${ }^{31}$ It has been postulated that delayed initiation of levothyroxine in pregnancy may account for its failure to reduce miscarriage. Our meta-analysis found that initiation of levothyroxine preconception or in the first trimester did not impact miscarriage outcomes. This suggests that inadequate thyroid reserve in women with thyroid autoimmunity does not account for the increased risk for miscarriage. A highly anticipated trial T4-LIFE that has not yet been published may further address the role of levothyroxine administration in women with TPOAb and recurrent miscarriage. ${ }^{32}$

Sensitivity analyses based on timing of levothyroxine initiation demonstrated a reduction in preterm delivery when levothyroxine was initiated in the first trimester rather than preconception. However, this finding is likely attributed to the quality of the included studies since many of the trials that initiated levothyroxine in the first trimester were at high risk of bias and the funnel plot suggests publication bias. Furthermore, it appears biologically implausible that levothyroxine would be beneficial for thyroid autoimmune women when initiated during pregnancy but not when initiated preconception.

A limitation of this study is that only one of the six included trials met all criteria for low risk of bias. Metaanalyses of live births and neonatal outcomes such as gestational age at delivery and birth weight were limited as only two trials reported these outcomes. ${ }^{89}$ Furthermore, the sensitivity analysis is limited by the small number of studies included. Lastly, the funnel plot suggests publication bias towards smaller studies reporting positive findings. Interpretation of the funnel plot is limited by the small number of studies included in this meta-analysis. ${ }^{33}$

This study has many notable strengths. First, this study provides an updated summary of randomised controlled trials not included in previous systematic reviews and meta-analyses or available at the time of the ATA 2017 pregnancy guideline recommendations development. The systematic review and meta-analysis 
provides moderate (GRADE) evidence for the preterm delivery and high (GRADE) evidence for the outcomes of pregnancy achieved, miscarriage and live birth to guide clinical decisions. Our systematic review and meta-analysis benefits from the use of rigorous standardised tools such as Cochrane Risk of Bias tool ${ }^{23}$ a prespecified analysis plan posted on Prospero, sensitivity analysis that address study quality and the assessment of publication bias.

\section{CONCLUSION}

In summary, data from six randomised controlled trials fail to demonstrate evidence of benefit from levothyroxine treatment for pregnancy outcomes in thyroid autoimmune women with normal thyroid function and subclinical hypothyroidism. In the absence of overt thyroid dysfunction, current trial evidence does not support the levothyroxine treatment of thyroid autoimmunity preconception or during the pregnancy. Given the lack of evidence of benefit of levothyroxine therapy, it is time to re-examine recommendations for screening $\mathrm{TPOAb}$ and treatment of women with thyroid autoimmunity preconception or in pregnancy.

\section{Author affiliations \\ ${ }^{1}$ Section of Endocrinology and Metabolism, Department of Medicine, Cumming \\ School of Medicine, University of Calgary, Calgary, Alberta, Canada \\ ${ }^{2}$ Department of Community Health Sciences, Cumming School of Medicine, University of Calgary, Calgary, Alberta, Canada \\ ${ }^{3}$ Department of Obstetrics and Gynecology, Cumming School of Medicine, University of Calgary, Calgary, Alberta, Canada \\ ${ }^{4}$ Alberta Children's Hospital Research Institute, Cumming School of Medicine, University of Calgary, Calgary, Alberta, Canada \\ ${ }^{5}$ Department of Medicine, University of Manitoba, Winnipeg, Manitoba, Canada}

Acknowledgements We thank Zahra Premji, PhD, MLIS (University of Calgary, Calgary, Alberta) for her expert advice on our search strategy.

Contributors PL and LED conceived the idea for this systematic review and metaanalysis, and conducted the literature search along with the initial title/abstract review. JY contributed her expertise in systematic review methodology to the design and conduct of this systematic review. LL and JLB conducted the full-text review, study selection, data extraction and statistical analyses. LL wrote the first draft of the manuscript. All authors contributed to critical review and approval of the final manuscript.

Funding The authors have not declared a specific grant for this research from any funding agency in the public, commercial or not-for-profit sectors.

Competing interests None declared.

Patient consent for publication Not required.

Ethics approval The authors adhered to the Preferred Reporting Items for Systemic Reviews and Meta-Analyses (PRISMA) guidelines. Ethical committee approval was not required for this study.

Provenance and peer review Not commissioned; externally peer reviewed.

Data availability statement All data relevant to the study are included in the article or uploaded as online supplemental information.

Supplemental material This content has been supplied by the author(s). It has not been vetted by BMJ Publishing Group Limited (BMJ) and may not have been peer-reviewed. Any opinions or recommendations discussed are solely those of the author(s) and are not endorsed by BMJ. BMJ disclaims all liability and responsibility arising from any reliance placed on the content. Where the content includes any translated material, BMJ does not warrant the accuracy and reliability of the translations (including but not limited to local regulations, clinical guidelines, terminology, drug names and drug dosages), and is not responsible for any error and/or omissions arising from translation and adaptation or otherwise.

Open access This is an open access article distributed in accordance with the Creative Commons Attribution Non Commercial (CC BY-NC 4.0) license, which permits others to distribute, remix, adapt, build upon this work non-commercially, and license their derivative works on different terms, provided the original work is properly cited, appropriate credit is given, any changes made indicated, and the use is non-commercial. See: http://creativecommons.org/licenses/by-nc/4.0/.

ORCID iD

Lorraine Lau http://orcid.org/0000-0001-9402-5425

\section{REFERENCES}

1 Moreno-Reyes R, Glinoer D, Van Oyen H, et al. High prevalence of thyroid disorders in pregnant women in a mildly iodine-deficient country: a population-based study. J Clin Endocrinol Metab 2013;98:3694-701

2 McElduff A, Morris J. Thyroid function tests and thyroid autoantibodies in an unselected population of women undergoing first trimester screening for aneuploidy. Aust N Z J Obstet Gynaecol 2008:48:478-80.

3 Pratt DE, Kaberlein G, Dudkiewicz A, et al. The association of antithyroid antibodies in euthyroid nonpregnant women with recurrent first trimester abortions in the next pregnancy. Fertil Steril 1993;60:1001-5.

4 Kutteh WH, Yetman DL, Carr AC, et al. Increased prevalence of antithyroid antibodies identified in women with recurrent pregnancy loss but not in women undergoing assisted reproduction. Fertil Steril 1999;71:843-8.

5 Thangaratinam S, Tan A, Knox E, et al. Association between thyroid autoantibodies and miscarriage and preterm birth: meta-analysis of evidence. BMJ 2011:342:d2616.

6 Consortium on Thyroid and Pregnancy-Study Group on Preterm Birth, Korevaar TIM, Derakhshan A, et al. Association of thyroid function test abnormalities and thyroid autoimmunity with preterm birth: a systematic review and meta-analysis. JAMA 2019;322:632-41.

7 Stagnaro-Green A, Roman SH, Cobin RH, et al. Detection of at-risk pregnancy by means of highly sensitive assays for thyroid autoantibodies. JAMA 1990;264:1422-5.

8 Dhillon-Smith RK, Middleton LJ, Sunner KK, et al. Levothyroxine in women with thyroid peroxidase antibodies before conception. $N$ Engl J Med 2019:380:1316-25.

9 Nazarpour S, Ramezani Tehrani F, Simbar M, et al. Effects of levothyroxine treatment on pregnancy outcomes in pregnant women with autoimmune thyroid disease. Eur J Endocrinol 2017;176:253-65.

10 Negro R, Formoso G, Mangieri T, et al. Levothyroxine treatment in euthyroid pregnant women with autoimmune thyroid disease: effects on obstetrical complications. J Clin Endocrinol Metab 2006;91:2587-91.

11 Negro R, Mangieri T, Coppola L, et al. Levothyroxine treatment in thyroid peroxidase antibody-positive women undergoing assisted reproduction technologies: a prospective study. Hum Reprod 2005;20:1529-33.

12 Negro R, Schwartz A, Stagnaro-Green A. Impact of levothyroxine in miscarriage and preterm delivery rates in first trimester thyroid antibody-positive women with TSH less than $2.5 \mathrm{mIU} / \mathrm{L}$. J Clin Endocrinol Metab 2016;101:3685-90.

13 Rao M, Zeng Z, Zhou F, et al. Effect of levothyroxine supplementation on pregnancy loss and preterm birth in women with subclinical hypothyroidism and thyroid autoimmunity: a systematic review and meta-analysis. Hum Reprod Update 2019;25:344-61.

14 Wang $\mathrm{H}$, Gao H, Chi H, et al. Effect of levothyroxine on miscarriage among women with normal thyroid function and thyroid autoimmunity undergoing in vitro fertilization and embryo transfer: a randomized clinical trial. JAMA 2017;318:2190-8.

15 Alexander EK, Pearce EN, Brent GA, et al. 2017 guidelines of the American thyroid association for the diagnosis and management of thyroid disease during pregnancy and the postpartum. Thyroid 2017;27:315-89.

16 Rao M, Zeng Z, Zhao S, et al. Effect of levothyroxine supplementation on pregnancy outcomes in women with subclinical hypothyroidism and thyroid autoimmuneity undergoing in vitro fertilization/intracytoplasmic sperm injection: an updated metaanalysis of randomized controlled trials. Reprod Biol Endocrinol 2018;16:92 
17 Sun $\mathrm{X}$, Hou N, Wang $\mathrm{H}$, et al. A meta-analysis of pregnancy outcomes with levothyroxine treatment in euthyroid women with thyroid autoimmunity. J Clin Endocrinol Metab 2020;105:1009-19.

18 Barton S. Which clinical studies provide the best evidence? The best RCT still trumps the best observational study. BMJ 2000;321:255-6.

19 HJ GS. Cochrane Handbook for Systematic Reviews of Interventions Version 5.1.0 [updated March 2011]. The Cochrance Collaboration, 2011.

20 Weeks I, Sturgess M, Siddle K, et al. A high sensitivity immunochemiluminometric assay for human thyrotrophin. Clin Endocrinol 1984;20:489-95.

21 Nicoloff JT, Spencer CA. Clinical review 12: the use and misuse of the sensitive thyrotropin assays. J Clin Endocrinol Metab 1990;71:553-8.

22 Cohen J. Weighted kappa: nominal scale agreement with provision for scaled disagreement or partial credit. Psychol Bull 1968;70:213-20.

23 Higgins JPT, Altman DG, Gøtzsche PC, et al. The Cochrane collaboration's tool for assessing risk of bias in randomised trials. BMJ 2011;343:d5928.

24 Guyatt GH, Oxman AD, Vist GE, et al. Grade: an emerging consensus on rating quality of evidence and strength of recommendations. $B M J$ 2008;336:924-6.

25 Higgins JPT, Thompson SG, Deeks JJ, et al. Measuring inconsistency in meta-analyses. BMJ 2003;327:557-60.
26 Casey BM, Thom EA, Peaceman AM, et al. Treatment of subclinical hypothyroidism or hypothyroxinemia in pregnancy. N Engl $J$ Med 2017;376:815-25.

27 Lazarus JH, Bestwick JP, Channon S, et al. Antenatal thyroid screening and childhood cognitive function. $N$ Engl $J$ Med 2012;366:493-501.

28 Cappola AR, Casey BM. Thyroid function test abnormalities during pregnancy. JAMA 2019;322:617-9.

29 Abdel Rahman AH, Aly Abbassy H, Abbassy AAE. Improved in vitro fertilization outcomes after treatment of subclinical hypothyroidism in infertile women. Endocr Pract 2010;16:792-7.

30 Wang $\mathrm{X}$, Zhang $\mathrm{Y}$, Tan $\mathrm{H}$, et al. Effect of levothyroxine on pregnancy outcomes in women with thyroid autoimmunity: a systematic review with meta-analysis of randomized controlled trials. Fertil Steril 2020;114:1306-14.

31 Glinoer DR M. [Thyroid changes in the pregnant woman]. Les alterations thyroidiennes chez la femme enceinte 1993;54:385-8.

32 Vissenberg R, van Dijk MM, Fliers E, et al. Effect of levothyroxine on live birth rate in euthyroid women with recurrent miscarriage and TPO antibodies (T4-LIFE study). Contemp Clin Trials 2015;44:134-8.

33 Sterne JAC, Sutton AJ, loannidis JPA, et al. Recommendations for examining and interpreting funnel plot asymmetry in meta-analyses of randomised controlled trials. BMJ 2011;343:d4002. 